\title{
Automated Framework for Segmenting Skin Lesions using Artificial Bee Colony Optimization with Morphological Reconstruction
}

\author{
R.Sumathi,M.Venkatesulu
}

\begin{abstract}
Nowadays, Many people are affected by skin cancers. Our proposed work designed a framework to extract the skin cancer using artificial bee colony with morphological reconstruction filters, which helps the demonologist to prevent the severity in early stage, Melanoma is the now become a harmful form of skin cancer which leads the skin cells to grow rapidly and form cancerous tumors. We collected various melanoma images from having used samples from public dataset like ISIC archive and a few from clinical datasets. To remove the noise, median filtering is used for preprocessing in the first step, to segment the tumor boundary Artificial bee colony is used and to remove the unwanted pixels using morphological reconstruction filters. Segmentation metrics like precision, recall, accuracy, Mean Square Error, Peak signal to noise ratio and computational time were calculated. Our proposed method yield $97.7 \%$ segmentation accuracy when compared with the level set method and Fuzzy C Means clustering techniques
\end{abstract}

Keywords :: Image Segmentation, Median Filter, Artificial Bee Colony Optimization, Morphological reconstruction filters,

\section{INTRODUCTION}

Image processing techniques are highly helpful in biomedical image processing, anaylzing, classifying the tumor and non tumor and clustering techiques for detecting and extracting the tumor part. Segmentation is used to segment the region os tumor part or detect the edge of tumor part by applying a variuos segmentation techniques. Melonoma looks and like a mole and it also develops from mole, which may caiuse skin cancer later times if it not analyzed in early stage, to reduce the death rate of skin cancer many researchers used soft computing techniques and clustering alorithms to segment the tumor part effeciently. Hence automated melanoma identification is necessary. we proposed Artificial Bee Colony(ABC) techniques used for identify the melanoma. This updating increased the slow convergence and thus helped to find the best solution for the algorithm. To extract

Revised Manuscript Received on December 16, 2019

R.Sumathi*, Department of Computer Science and Engineering, Kalasalingam Academy of Research and Education, Tamil Nadu, India. Email: suchandika@gmail.com

M.Venkatesulu, Department of Information Technology, Kalasalingam Academy of Research and Education, Tamil Nadu, India

Email: venkatesulu2000m@gmail.com the tumor features, it is important to estimate the accurate lesion border which is used for segmentation and classification. Its aim is to segment the tumor part accuracy with less time with efficient results.

To segment the exact shape of skin lesions using semi automatic approach[1] and classify the skin lesions of dermoscopy image dataset.[2] Applying morphological filters and neural network to classify the skin lesions with benign or malignant and ensure its accuracy. [3] Classify the tumor and non tumor by applying the threshold based segmentation and proved that joint statistical texture distinctiveness method leads greater segmentation accuracy . Color lesion segmentation based on Decision Based Neuro Fuzzy Model and segments the skin lesions based on color texture and its limitations are when the color space format changed to other format depends on usability for various kinds of skin cancer images[4]. To detect the skin lesions genetic algorithm is used to segment the tumor part with short duration for different scales and levels of qualities of skin lesion images and reduce the over segmentation[5]. Applying wavelet base networks to segment the dermoscopy images based on four methods like AT, GVF, FBSM, and NN[6]. An automated segmentatio model is developmed with the combination of SGNN and GA for segmenting the lesions of dermoscopy images with neural network feature set [7]. An automated border detection method is used to extract the pigmented skin lesions using statistical region merging techniques and validate the method with 90 images with ground truth images and proved their accuracy[8]. Combining artificial neural network and gray wolf optimizer[9] produces $90 \%$ of classification accuracy and proved that it can able to detect skin malignant melanoma images better than ANN classifier.Skin lesions are segmented and classified using Conventional neural network[10] and it yield 96\% of classification and proved its efficiency. An hybrid approach with the Combination of PSO and GWO [11] proved its efficiency with best features obtained from the two methods 


\section{Automated Framework for Segmenting Skin Lesions using Artificial Bee Colony Optimization with Morphological Reconstruction}

\section{METHODS AND MATERIALS}

\subsection{Scope of our work}

Our goal is to segment the tumor part of skin dieases by applying Arrtificial ant bee colony with morphological reconstruction filters. This automated method is highly used by dermologist to analyze the tumor growth in advance and reduce the growth of tumor in advance . we proved that our method produce accurate segmentation than level set and FCM method in terms of accuracy and computation time

\subsection{Details of Image datasets}

For our study and analyze the meloname skin cancer images collected form online free datasets like TCIA, dermis dataset and few from clinical datasets for proving the efficiency of our methodology.

\subsection{Image Analysis}

The images were analyzed using the following steps Step 1: Read all types of melanoma images and rezise the images into $256 * 256$ grayscale images .

Step 2: Preprocess the image by applying the median filters to enhance the details of tumor part for segmentation

Step 3: To segment the boundary of tumor part by applying the artificial bee colony

Step 4: To remove the unrelavant pixels near the tumor by appying otsu threshoding method to segment the auurate tumor part. Figure 1 describes the descriptions of our proposed work.

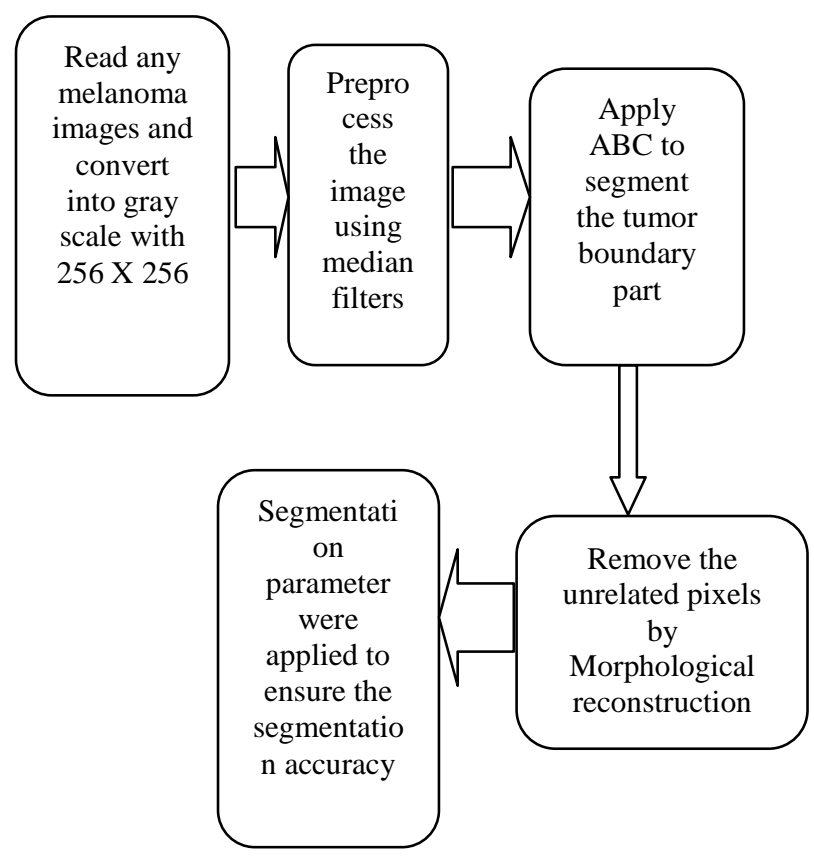

Figure 1 : Flow diagram of our proposed work

\subsection{Median Filtering}

One of the enhancement techniques which removes the impulse noise without reducing the image sharpness [12] where as $\mathrm{A}(\mathrm{m}, \mathrm{n})$ is the median filtered image and $\mathrm{g}(\mathrm{x}, \mathrm{y})$ is the input image

\subsection{Artificial Bee Colony}

Artificial bee colony is a famous method which replicates the perspective actions of honey bees. It was divided into two branches like worker bee and onlooker bees, whereas the first half of thegroup is accupied by worker bee and the remaining part is occupied by the onlooker bees[13].The preproessed image is given to $\mathrm{ABC}$ for segmenting the boundary of tumor with random intial population based on th number os worker bee food resources generated by the $\mathrm{ABC}$

Let $Y_{i=}\left[Y_{i, 1}, Y_{i, 2}, \ldots \ldots . Y_{i, N}\right]$ denotes the $\mathrm{i}^{\text {th }}$ set of solution in the group, whereas size of the bees represented by N. Each worker bee- $Y_{\mathrm{i}}$ creates a new resoultion $\mathrm{Z}_{\mathrm{i}}$ in the locality of its nearby position is represent ed as

$Z_{i, j}=y_{i, j}+\sigma_{i, j}\left(y_{i, j}-y_{k, j}\right)(2)$

Where $\mathrm{y}_{\mathrm{k}}$ represents the elected resolution, $\mathrm{J}$ represents the length index, the value of $\sigma_{i, j}$ lies between -1 to +1 . Check the value of $\mathrm{Z}_{\mathrm{i}}$, if it is self-satisfied than old $Y_{\mathrm{i}}$, then revision of $Y_{\mathrm{I}}$ with $\mathrm{Z}_{\mathrm{i}}$, else the value of $\mathrm{y}_{\mathrm{i}}$ is fixed. when the search task is finished by all $Y_{\mathrm{i}}$ bees, and shares the value to onlooker bees and then onlooker bee calcuates the nectar and selects the best resource among all the possibilities.

Probability selection $\left(\mathrm{P}_{\mathrm{i}}\right)$ is defined as

$$
P_{i}=\frac{F_{i}}{\sum_{j=1}^{S R} F_{i}}(3)
$$

Where $F_{i}$ represents the fitnessamount for $i^{\text {th }}$ persistence within the group. Suppose that the discarded source is $Y_{i}$, the scout bee determines a fresh edible material resource to reinstate with $\mathrm{Y}_{\mathrm{i}}$ by using

$y_{i, j}=L B_{i, j}+r\left(U B_{i, j}-L B_{i, j}\right)$

Where $r$ lies between 0 to $1, \mathrm{UB}$ and LB defines the minimum and maximum sizes[14]

The following steps explain the algorithm in detail

1. initialize a random set of bees.

2. Using the initial swarm renew the solution.

3. Equation (2) is helped to find the new candidate value of $\mathrm{Z}_{\mathrm{i}}$ and also find the fitness function based on equation (2)

4. Every onlooker bee evaluates $P_{i}$ based on Equation (3).

5. Generate a new candidate resolution $Z_{i}$ based on Equation

(2) and Find the fitness of $Z_{i}$ and a hunger selection is utilized to select a best one between $\mathrm{Y}_{\mathrm{i}}$ and $\mathrm{Z}_{\mathrm{i}}$ like the new $\mathrm{Y}_{\mathrm{i}}$.

6. unwanted bees are updated using the equation (4)

7.Update the superlative resolution institute until it met the stoping condition ,

and update the value of iter $=$ iter +1 .

8. check the value of iter, if it exceeds the specified number of steps then stop and display the output or else goto step 2 9: end

$\mathrm{A}(\mathrm{m}, \mathrm{n})=\operatorname{median}\{\mathrm{g}(\mathrm{x}, \mathrm{y})\}$ 


\subsection{Morphological Reconstruction Filters}

It is a useful tool for medical image segmentation, image analysis and pattern recognition. To extract the exact shape of the tumor morphological reconstruction filters are preferrable. various operations like erosion, dilation, opening and closing are defined as [15]

$$
\begin{aligned}
& \text { Erosion : } A \ominus B=\{x \mid B+x \subseteq A\} \\
& \text { Dialtion : } A \oplus B=\{x \mid B+x \neq \emptyset\} \\
& \text { Opening : } A \circ B=\{A \ominus B\} \oplus B(7) \\
& \text { Closing : } A \cdot B=\{A \oplus B\} \ominus B
\end{aligned}
$$

For segmening the accurate tumor part

$\beta(A)=A-(A \ominus B)$

A represents the input image with structural element $B$.

\section{RESULTS AND DISCUSSIONS}

Segmentation metrics like precision, recall, MSE, PSNR, accuracy and computation time are measured to ensure accuracy of our proposed work. It was implemented by using MATLAB 2017a software. The Mean square error is denoted by

$M S E=\frac{\sum A, B\left[x_{1}(a, b)-x_{2}(a, b)\right]^{2}}{A * B}$

where $\mathrm{A}$ and $\mathrm{B}$ provides the size of row and column for the input image, whereas PSNR can be calculated by

$P S N R=10 \log _{10}\left(\frac{R^{2}}{M S E}\right)$

Where $\mathrm{R}$ represents the maximum fluctutation value of the given imput image. It measures the quality of noise information between the input and segmented image computational time is measured between the given image and extracted output thyroid gland image. Recall measures the area of thyroid gland from the total area of the image

Recall $=\frac{T_{p}}{T_{p}+F_{n}}$

Precision calculates the background ratio from the total area of the background

Precision $=\frac{T_{p}}{T_{p}+F_{p}}$

Accuracy ensures the segmentation rate between the manual segmentation and expert segmentation

Accuracy $=\frac{T_{p}+T_{n}}{T_{p}+T_{n}+F_{p}+F_{n}}$

Our proposed work ensures the segmentation accuracy using various segmentation metrics and also compared with computational time with other existing approach for segmenting the skin cancer tumor in less duration. Table 1 details the MSE and PSNR measures of our proposed method. Mean square error value is lies between 0-1ensures the less noise ratio and PSNR default value lies between 40 -60 decibels ensures the high quality with less noise signals.

Table 1: performance Measure of our proposed method

\begin{tabular}{|c|c|c|}
\hline $\begin{array}{c}\text { Image } \\
\text { No }\end{array}$ & MSE & PSNR \\
\hline 1 & 0.009 & 53.46 \\
\hline 2 & 0.007 & 48.93 \\
\hline 3 & 0.003 & 55.73 \\
\hline 4 & 0.005 & 50.08 \\
\hline 5 & 0.004 & 49.67 \\
\hline 6 & 0.002 & 51.04 \\
\hline 7 & 0.007 & 47.05 \\
\hline 8 & 0.006 & 46.85 \\
\hline Avg & $\mathbf{0 . 0 0 5}$ & $\mathbf{5 0 . 3 5 1}$ \\
\hline
\end{tabular}

Our proposed work yield overall $50.35 \%$ of PSNR and $0.005 \%$ of MSE whereas FCM produces 32\% PSNR and $1.33 \%$ MSE and level set produces 37\% PSNR and 1.34\% MSE values. The quantitative comparison among the existing methods was given in Table 2.

Table 2: Segmentation Metrics of our proposed method

\begin{tabular}{|c|c|c|}
\hline Image No & Accuracy & $\begin{array}{c}\text { Time } \\
\text { Consumption (s) }\end{array}$ \\
\hline 1 & 97.5 & 8.33 \\
\hline 2 & 98.5 & 8.43 \\
\hline 3 & 97.77 & 7.44 \\
\hline 4 & 97.21 & 8.47 \\
\hline 5 & 99.52 & 8.4 \\
\hline 6 & 98.22 & 6.72 \\
\hline 7 & 97.31 & 6.82 \\
\hline 8 & 97.76 & 7.89 \\
\hline
\end{tabular}

Recall ensures the quality of the tumor segmentation, whereas accuracy measures ensure the efficiency of skin tumor images with ground truth image segmentation. Time takes for segmenting the tumor is also mentioned in table 2.Computational time among other techniques are compared with our proposed work is mentioned in Table 3 .

Table 3 :Comparative Measures of proposed with existing method

\begin{tabular}{|c|c|c|}
\hline Methods & Accuracy & $\begin{array}{c}\text { Time Consumption } \\
(\mathrm{s})\end{array}$ \\
\hline FCM & 94.5 & 45 \\
\hline Level set & 93.6 & 12 \\
\hline Proposed & 97.5 & 7 \\
\hline
\end{tabular}

Published By: 


\section{Automated Framework for Segmenting Skin Lesions using Artificial Bee Colony Optimization with Morphological Reconstruction}

It was proved that our $\mathrm{ABC}$ with morphological reconstruction filters is highly recommended our skin lesions segmentation in near future. A structural element is more useful for removing the extraneous pixels surrounded with tumor part. we used 'disc' as the structural element with $9 \mathrm{X}$ 9 value for all images used in our study.
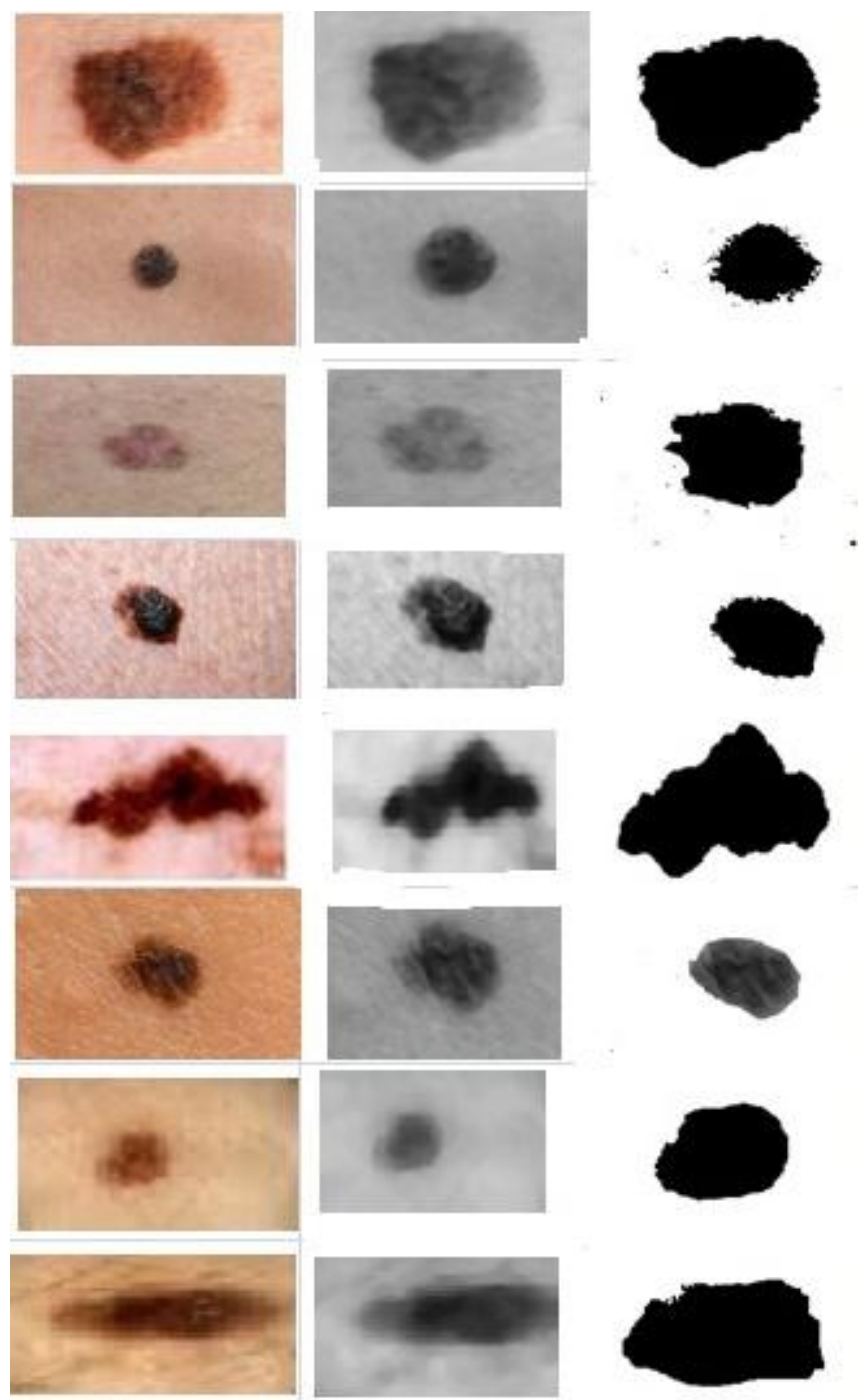

Figure 2 : Melanoma Skin Images collected from Iris and Clinical dataset Figure 2a) input images 2b) contains Median Filtered Image and 2c) contains $\mathrm{ABC}$ with morphological reconstruction filtered images

Figure $2 \mathrm{a}$ contains original input image and figure $2 \mathrm{~b}$ contain the median filtered image and Figure $2 \mathrm{c}$ contains $\mathrm{ABC}$ with morphological reconstruction filter images of our proposed work.
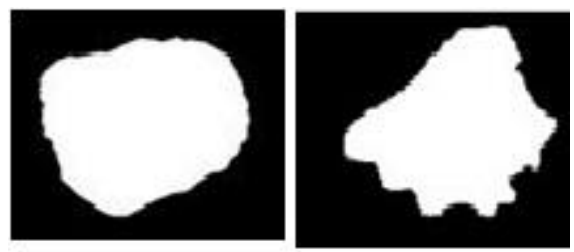

Figure 3 :Ground truth images of skin tumor

Figure 3 contains sample ground truth images of melanoma skin cancer images collected from IRIS dataset .

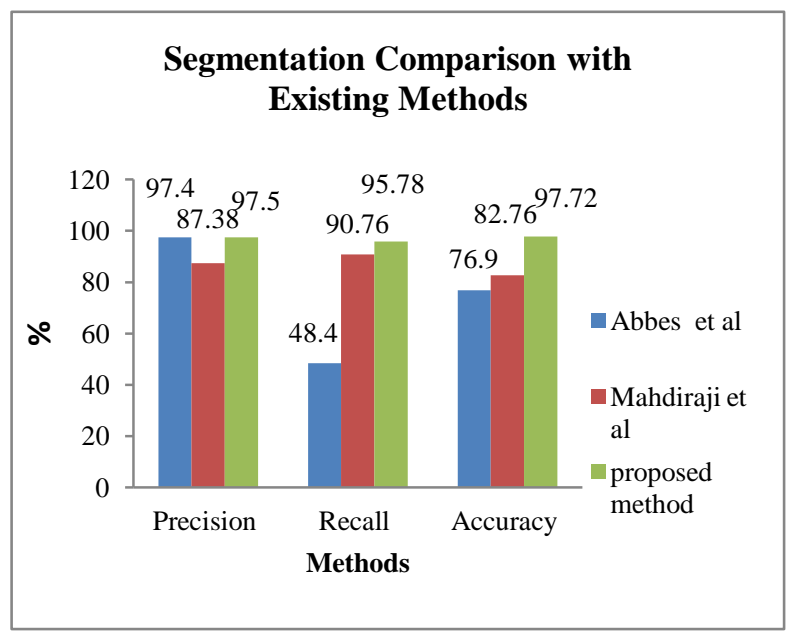

Figure 4: Comparison with other Existing Techniques

Figure 4 compares the performance of segmentation with other existing approaches [16-17]. it was ensured that the proposed work produces best segmentation than the existing approaches also it compares the accuracy measures with state of art

\section{CONCLUSION}

We used an automated design using artificial bee colony with morphological reconstruction filters to segment the skin lesions within short duration. This design is more helpful for the demonologist for early detection and prevention of tumor growth in advance. The segmentation performance were evaluated and compared the computational time with other state of art methods. It was noticed that some images produces incorrect segmentation and additional pixels are along with the tumor, that limitations are overtaken by the morphological reconstruction filters

\section{REFERENCES}

1. Zhen Ma and Joao Manuel R. S. Tavares," A Novel Approach to Segment Skin Lesions in Dermoscopic Images Based on a Deformable Model", IEEE Journal of Biomedical, 2016, Vol.20, pp7132-7138

2. M.Yuvaraju, D.Divya, A.Poornima, "Segmentation of skin lesion from digital images using morphological filter", IEEE, 2016, Vol.3, pp 2395-0072

3. Revathi V.L, Chithra," A review on segmentation techniques in skin lesion images", International Research Journal,2015, Vol. 2,pp. 2395-0072.

4. BinamrataBaral, SandeepGonnade, ," Lesion Segmentation in Dermoscopic Images Using Decision Based Neuro Fuzzy Model",International Journal of Computer Science and Information Technologies,2014,Vol. 5,pp 2546- 2552

5. HindaSood, ManshiShukla," An approach for detection of ski $\mathrm{n}$ lesions from digital images using genetic algorithm", International Journal of Computer science and Information Technology, 2014, Vol.5,pp. 6831-6837
Maryam Zekri, Saeed Sadri, Insofar, "Segmentation of Dermoscopy Images Using Wavelet Networks", IEEE 
Transaction on Biomedical Engineering, 2013, Vol. 60, pp.1134-41

7. Xie, F, Fan, H.; Li, Y, Jiang, Z, Meng, R, Bovik. A, “ Melanoma classification on dermoscopy images using a neural network ensemble model", IEEE Transaction on Medical Imaging, 2017, Vol.36, pp $849-858$

8. Celebi M E, Kingravi H A, Iyatomi H, Aslandogan Y A, Stoecker W V, Moss R H, Malters J M, Marghoob A A, Rabinovitz H S, Menzies S $\mathrm{W}$, Border detection in demoscopy images using statistical region merging”, Skin Res Technology, 2008, Vol.14, No.3,pp 347-53

9. Ali Parsian, Mehdi Ramezani and NoradinGhadimi, "A hybrid neural network grey wolf optimization for melanoma detection", Biomedical Research, 2017, Vol.28, pp. $3408-3411$

10. Jadhav A.R., Ghontale A.G, Shrivastava V.K, "Segmentation and Border Detection of Melanoma Lesions Using Convolution Neural Network and SVM", Advances in intelligent systems and Computing, 2019, Vol.1, pp.97-108

11. Yogendra Singh Kushwah, R.K. Shrivastava, "Particle Swarm Optimization Inspired Grey Wolf Optimization Algorithm", International Journal of Mathematics Trends and Technology, 2018, Vol.58, pp 2,135-149

12. Sumathi R, Arjunan S, “ Towards better segmentation of object of interest using histogram equalization and morphological reconstruction". International Journal Signal Imaging System Engineering, 2014, Vol. 7,pp 189-94

13. Karaboga, D."An Idea Based on Honey Bee Swarm for Numerical Optimization", Technical Report-tr06. Erciyes University. Engineering Faculty, Computer Engineering Department: Kayseri, Turkey, 2005.

14. Barata. C, Ruela. M, Francisco. M, Mendonça T. Marques. J.S,” Two systems for detection of melanomas in ceroscopy images using texture and color features", IEEE Syst. J,2014, Vol. 8, pp 965-979

15. Gonzalez R.C, Woods R.E. "Digital image processing". $3^{\text {rd }}$ Edition, New Jersey, Prentice Hall, Incorporation, 2009.

16. Mahdiraji, S.A., Baleghi, Y, Sakhaei, S.M. "Skin Lesion Images Classification Using New Color Pigmented Boundary Descriptors", In Proceedings of the 2017, $3^{\text {rd }}$ international conference on pattern recognition ton and image analysis,(IPRIA), Iran, pp102-107

17. Abbas W, Sellami D, "Automatic skin lesions classification using ontology based semantic analysis of optical standard images". Procedia Computer Science, 2017, Vol. 112, pp 2096-2105 